\title{
Etiología de la neumonía adquirida en la comunidad en adultos hospitalizados en Santiago, Chile: implicancias para las guías clínicas
}

\author{
ALEJANDRO DÍAZ F.*, GINO FUENTES L.****, BERNARDITA COUBLE P.****, \\ REINALDO URIBE S.M.***, GESMA MERCADO M.*, ALEJANDRA SOZA G.**, \\ PAULINA BARRÍA P.*, JORGE DREYSE D.*** y FERNANDO SALDÍAS P.* \\ ETIOLOGY OF COMMUNITY ACQUIRED PNEUMONIA REQUIRING \\ HOSPITALIZATION IN CHILE: IMPLICATIONS FOR THE GUIDELINES
}

Background: There are a few studies examining the etiology of community acquired pneumonia (CAP) in Chile. Aim: To evaluate the etiology of CAP in hospitalized adults. Method: We prospectively studied 130 patients (mean age $\pm S D$ : $68 \pm 18$ y.o.; overall hospital mortality: 6.2\%), over a 16 month period. Microbiological evaluation included blood and sputum cultures for bacteria; serology for $\boldsymbol{C}$. pneumoniae, $\boldsymbol{C}$. psittaci and $\boldsymbol{M}$. pneumoniae; urine antigen for $\boldsymbol{L}$. pneumophila; and nasopharyngeal swab for respiratory viruses. Results: Etiology was identified from 64 (49\%) patients (two or more pathogens in 6). The most frequent microorganisms were $\boldsymbol{S}$. pneumoniae (34\%), Parainfluenza types 1 to 3 (22\%), Influenza A or B (14\%), C. pneumoniae (6\%), M. pneumoniae (6\%), H. influenzae (5\%) and S. marcescens (5\%). Twenty-five of 27 (93\%) respiratory viruses were identified in autumn or winter. Pneumococcal pneumonia patients (19) compared to those infected with respiratory virus (23) were younger $(59 \pm 18$ versus $72 \pm 17$ y.o.; $p=0.021$ ) and had less comorbidities (47 versus 87\%; $p=0.0001$ ). No patients with bacteremia (13 of 121: 11\%) died. Conclusions: S. pneumoniae remains the most important pathogen to cover by initial antibiotic therapy; the second most frequent etiological agents were respiratory viruses followed by "atypical pathogens". Recommendations for the management of patients infected with these two last categories of agents should be included in future national guidelines.

Key words: community acquired pneumonia hospitalized, etiology, guidelines, virus, bacterial.

\section{RESUMEN}

Fundamento: Hay escasos estudios que examinen la etiología de la neumonía adquirida en la comunidad (NAC) en población adulta chilena. Objetivo: Identificar la etiología de la NAC en adultos inmunocompetentes hospitalizados. Método: Estudiamos, prospectiva y consecutivamente durante 16 meses, a 130 pacientes (edad promedio \pm DS: $68 \pm 18$ años; letalidad en el hospital: 6,2\%). La evaluación microbiológica incluyó cultivo de expectoración y hemocultivos para bacterias;

\footnotetext{
* Departamento de Enfermedades Respiratorias, Pontificia Universidad Católica de Chile.

** Servicio de Urgencias, Pontificia Universidad Católica de Chile.

*** Estudiantes de Medicina de tercer año de la Pontificia Universidad Católica de Chile.

**** Estudiantes de Medicina de cuarto año de la Pontificia Universidad Católica de Chile.
}

Financiamiento: Fondo de Investigación de la Sociedad Chilena de Enfermedades Respiratorias (2002) y fondo de la Dirección de Investigación de la Pontificia Universidad Católica de Chile (DIPUC 2003/10E). 
serología para $C$. pneumoniae, $C$. psittaci, $M$. pneumoniae; antígeno urinario para L. pneumophila e hisopado nasofaríngeo para virus respiratorios. Resultados: Se identificó la etiología en 64 (49\%) pacientes (dos o más patógenos en 6). Los principales microorganismos fueron: S. pneumoniae (34\%), virus Parainfluenza 1 a 3 (22\%), virus Influenza $A$ o B (14\%), C. pneumoniae (6\%), M. pneumoniae (6\%), H. influenzae (5\%) y S. marcescens (5\%). El 93\% (25/27) de los virus respiratorios se identificaron en otoño-invierno. Los pacientes con neumonía neumocócica (19) comparados con aquéllos infectados por virus respiratorios (23) eran más jóvenes $(59 \pm 18$ versus $72 \pm 17$ años; $p=0,021)$ y tenían menos comorbilidades (47\% versus 87\%; $p=0,0001)$. Ninguno de los 13 (11\%) pacientes con bacteremia falleció en el hospital. Conclusiones: $\boldsymbol{S}$. pneumoniae sigue siendo el principal patógeno a cubrir por el tratamiento antibiótico empírico; los virus respiratorios y los "agentes atípicos" fueron los que siguieron en frecuencia. Las futuras guías clínicas nacionales deberían incluir recomendaciones para el manejo de los pacientes infectados por estos dos últimos grupos de agentes etiológicos.

Palabras clave: neumonía adquirida en la comunidad; recomendaciones; etiología; bacteriana; viral.

\section{INTRODUCCIÓN}

La neumonía es la tercera causa específica de muerte en la población chilena y comprende casi la mitad de los egresos hospitalarios por enfermedades respiratorias. En 2001, la tasa de mortalidad fue 28 por 100.000 habitantes y 2,28 por 1000 en los mayores de 70 años ${ }^{1}$. Según el lugar de hospitalización, la letalidad de la neumonía adquirida en la comunidad (NAC) fluctúa entre 3 y 17\% $\%^{2}$. El 97\% de los casos comunicados al Ministerio de Salud es de etiología desconocida ${ }^{1}$, pero esta enfermedad no es de notificación obligatoria y el agente causal no siempre se consigna, por lo que esta cifra puede estar sobredimensionada.

Se reconoce que la información que brindan los estudios etiológicos de la NAC es uno de los pilares para realizar las recomendaciones de tratamiento antibiótico empírico por parte de diferentes Sociedades Médicas ${ }^{3-5}$; en estudios de otros países, S. pneumoniae es el patógeno principal, siendo la causa en el 36 a $62 \%$ de los episodios $^{6-14}$. La frecuencia e importancia de otros agentes etiológicos varía según el lugar geográfico, la época de realización de la investigación, la población estudiada y los métodos diagnósticos utilizados en el estudio ${ }^{15,16}$. En Chile, los estudios de la etiología de la NAC en población adulta se enfocaron en algún patógeno en particular ${ }^{17-19}$, en otros no se incluyeron los virus o los agentes atípicos sistemáticamente ${ }^{2}$ $\mathrm{y}$, cuando se incluyeron estos últimos, el tamaño muestral fue pequeño ${ }^{20}$.

De acuerdo a nuestro conocimiento, no se ha publicado un estudio etiológico que incluya la mayoría de los microorganismos causales de la
NAC en población adulta en Chile. El objetivo del presente trabajo fue evaluar la etiología de la neumonía adquirida en la comunidad en la población adulta inmunocompetente que requirió hospitalización para determinar la frecuencia e importancia de los diferentes patógenos causales.

\section{PACIENTES Y MÉTODO}

Se evaluaron prospectiva y consecutivamente los pacientes mayores de 14 años inmunocompetentes hospitalizados por NAC desde Marzo de 2003 a Junio de 2004 en el Hospital Clínico de la Pontificia Universidad Católica de Chile. El episodio de neumonía se definió, de acuerdo a los criterios descritos por Fang y cols ${ }^{8}$, como una enfermedad respiratoria aguda asociada a un infiltrado nuevo y persistente en la radiografía de tórax. Los criterios de exclusión fueron: serología positiva para el virus de la inmunodeficiencia humana (VIH), neoplasia activa, neutropenia (recuento de leucocitos $<1.000$ por $\mathrm{mm}^{3}$ ), tratamiento quimioterápico o inmunosupresor (ciclosporina, azatioprina) en los 6 meses previos al ingreso, uso de $20 \mathrm{mg}$ /día de prednisona durante el mes anterior y hospitalización por cualquier causa dentro de los 30 días previos al ingreso. Los pacientes fueron eliminados si el diagnóstico final del episodio no fue neumonía.

Evaluación clínica y de laboratorio: Al ingreso se registraron los siguientes antecedentes clínicos: edad, sexo, presencia de comorbilidades, hábito tabáquico y consumo de alcohol, lugar de procedencia (hogar, centro geriátrico u otro hospital) y cuadro clínico de presentación. La gravedad y el riesgo de muerte al ingreso fueron 
evaluados con el índice de gravedad de la neumonía (en inglés sigla PSI) descrito por Fine y cols $^{21}$. También se registró el lugar de ingreso (sala, unidad de intermedio o unidad de cuidados intensivos [UCI]), los signos vitales, la saturación arterial medida por oxímetro de pulso y los exámenes de laboratorio de admisión al hospital: gases arteriales, hemograma, electrolitos plasmáticos, glicemia, creatinina y nitrógeno ureico.

Radiografía de tórax: Los hallazgos de la radiografía de ingreso se clasificaron como patrón de relleno alveolar, intersticial o mixto. La extensión del compromiso se clasificó en unilobar, multilobar ( $\geq 2$ lóbulos) o bilateral y se registró la presencia de derrame pleural y excavación.

Evaluación microbiológica: A todos los pacientes incluidos se les tomó en el Servicio de Urgencias o en el lugar de hospitalización y, antes que se iniciara el tratamiento antibiótico, los siguientes exámenes: a) Dos hemocultivos aeróbicos. Las botellas se incubaron en el sistema automatizado de hemocultivos BacT/Alert 3D ${ }^{\circledR}$ (Compañía bioMérieux, Missouri, EE.UU.) $\mathrm{y}$, en aquellas en que el equipo detectó crecimiento, se realizó tinción de Gram y resiembra en agar sangre de cordero, agar chocolate y Mac Conkey. La identificación y los estudios de susceptibilidad se realizaron según métodos convencionales. Para definir los puntos de corte de susceptibilidad en Streptococcus pneumoniae aislados de infecciones respiratorias se utilizaron las normas del Comité Nacional de Estandarización de Laboratorios Clínicos (NCCLS) de EE.UU. b) Muestra de expectoración para Gram y cultivo aeróbico corriente. Se consideró que la muestra era de buena calidad cuando tenía menos de 10 células epiteliales y más de 25 leucocitos por campo de mayor aumento y sólo éstas se sembraron para cultivo. c) Muestra de sangre para serología (ingreso y a 30 días) de: 1) Mycoplasma pneumoniae: determinación de $\operatorname{IgM}$ e IgG por técnica de inmunofluorescencia indirecta (Compañía Zeus Cientific ${ }^{\circledR}$, New Jersey, EE.UU.); 2) Chlamydia pneumoniae y Chlamydia psittaci: determinación de $\operatorname{IgM}$ e $\operatorname{IgG}$ por técnica de microinmunofluorescencia (Compañía Vircell, Granada, España); d) Muestra de hisopado nasofaríngeo para la búsqueda de los virus Influenza A y B, Parainfluenza 1, 2 y 3, Adenovirus y Virus Sincicial Respiratorio. Se empleó la técnica de inmunofluorescencia directa (PathoDx Respiratory Virus Panel ${ }^{\circledR}$, Compañía Remel, Kansas, EE.UU.); e) Muestra de orina para la búsqueda del antígeno de Legionella pneumophila serotipo 1 por técnica de ELISA (Binax Now ${ }^{\circledR}$, Compañía Binax, Maine, EE.UU.).

Criterios de diagnóstico etiológico: Los criterios de diagnóstico etiológico considerados definitivos, según se ha descrito antes ${ }^{8,12}$, fueron: A) Aislamiento de un patógeno en un hemocultivo o líquido pleural. Staphylococcus coagulasa negativo y los bacilos Gram positivos difteromorfos se consideraron contaminantes; B) Antígeno urinario positivo para Legionella pneumophila serotipo 1; C) Aumento $\geq 4$ veces del título de IgG (fase aguda y convalescencia) para $C$. pneumoniae, C. psittaci y M. pneumoniae. Se consideró que el diagnóstico fue presuntivo cuando se cumplen los siguientes criterios: A) Crecimiento regular o abundante de un patógeno bacteriano en expectoración concordante con el Gram; B) Título único de $\operatorname{IgM} \geq 1 / 32$ para $C$. pneumoniae o $M$. pneumoniae (modificado de referencias 12 y 13); C) Inmunofluorescencia directa positiva para Influenza A y B, Parainfluenza 1 a 3, Adenovirus y Virus Sincicial Respiratorio en hisopado nasofaríngeo.

Tratamiento antibiótico y seguimiento: Se registraron los antibióticos empíricos prescritos, los traslados de una unidad a otra y entre las 3 a 6 semanas del diagnóstico los pacientes fueron citados para tomarles la segunda muestra de sangre del par serológico. Se registró la letalidad en el hospital.

El protocolo fue aprobado por el Comité de Ética del hospital y los pacientes firmaron un consentimiento informado al ingreso.

Análisis de los resultados y estadística: $\mathrm{La}$ principal variable medida fue la proporción de pacientes en los que se encontró un agente causal y el tipo de patógenos. En el subgrupo de pacientes con etiología demostrada se evaluó si el tratamiento empírico cubrió los patógenos correctamente. El tratamiento se definió como adecuado si al menos un antibiótico prescrito dentro de las primeras 24 horas de ingreso tenía actividad contra el patógeno aislado.

Para las variables categóricas se utilizó el test de $\chi^{2}$ y cuando correspondía el test de Fisher y para las variables continuas se usó el test $t$ de Student. Un valor de $\mathrm{p}<0,05$ se consideró significativo.

\section{RESULTADOS}

Se enrolaron 176 pacientes, pero 46 se eliminaron por las siguientes razones: ausencia de infiltrado en la radiografía de tórax (34), otros 
diagnósticos (9) y hospitalización previa (3). La población final de estudio comprendió 130 pacientes. La edad promedio ( \pm DS) fue $68 \pm 18$ años (rango 17 a 101), el 51\% era del sexo masculino y el $71 \%$ tenía enfermedad previa, siendo las más frecuentes cardiovasculares $(29 \%)$, EPOC o asma (21\%) y Diabetes mellitus (20\%).

Hallazgos clínicos: Las características clínicas y letalidad (Tabla 1) fueron semejantes en los pacientes con etiología conocida o desconocida, pero los primeros tenían menos edad $(64 \pm 21$ versus $71 \pm 15$ años; $p=0,03)$ y los síntomas antes del ingreso duraron en promedio 2 días más $(8 \pm 7$ versus $6 \pm 4$ días; $p=0,047)$.

Hallazgos de laboratorio y radiográficos: Los hallazgos radiográficos y de laboratorio fue- ron semejantes en los pacientes con etiología conocida y desconocida (Tabla 2).

Etiología: Al menos un agente etiológico fue identificado en 64 pacientes (49\%) y en 6 de ellos dos o más. En la Figura 1 se muestra la distribución temporal del total de casos y los causados por $S$. pneumoniae y virus respiratorios. En $16(72 \%)$ de 22 pacientes infectados con neumococo el agente se aisló en otoñoinvierno comparado con 25 (93\%) de 27 infectados con un agente viral $(\mathrm{p}=0,12)$. El porcentaje de pacientes con etiología identificada fue decreciendo significativamente con la edad: 14 a 40 años (12/15: 80\%), 41 a 65 años (20/38: 53\%) y mayores de 65 años (32/77: 41\%; $\mathrm{p}=0,02)$. Entre los 40 pacientes que recibieron un antibió-

Tabla 1. Características clínicas en 130 pacientes hospitalizados por neumonía adquirida en la comunidad

\begin{tabular}{|c|c|c|}
\hline Hallazgos Clínicos & $\begin{array}{c}\text { Pacientes con etiología } \\
\text { identificada }\end{array}$ & $\begin{array}{l}\text { Pacientes sin etiología } \\
\text { identificada }\end{array}$ \\
\hline $\mathrm{n}$ & 64 & 66 \\
\hline Edad, años (promedio \pm DS) & $64 \pm 21$ & $71 \pm 15^{*}$ \\
\hline Procedencia de geriátrico, n/total (\%) & $2 / 63(3 \%)$ & $2 / 66(3 \%)$ \\
\hline Días de síntomas antes ingreso (promedio \pm DS) & $8 \pm 7$ días & $6 \pm 4$ días* \\
\hline Lugar de ingreso & $\mathrm{n} /$ total $(\%)$ & $\mathrm{n} /$ total $(\%)$ \\
\hline Sala & $42 / 64(65)$ & $46 / 66(70)$ \\
\hline Intermedio & $10 / 64(16)$ & $13 / 66(20)$ \\
\hline Intensivo & $12 / 64(19)$ & $7 / 66(10)$ \\
\hline Comorbilidades & $44 / 64(69)$ & $48 / 66(73)$ \\
\hline Diabetes Mellitus & $12 / 64(19)$ & $14 / 66(21)$ \\
\hline Cardiopatía coronaria & $6 / 64(9)$ & $13 / 66(20)$ \\
\hline Arritmia crónica & $10 / 64(16)$ & $10 / 66(15)$ \\
\hline EPOC & $10 / 64(16)$ & $10 / 66(15)$ \\
\hline Demencia & $4 / 64 \quad(6)$ & 9/66 (14) \\
\hline Uso antibiótico previo al ingreso & $19 / 61(31)$ & $21 / 66(32)$ \\
\hline Compromiso de conciencia & $7 / 64(11)$ & $11 / 66(17)$ \\
\hline Calofríos & $29 / 64(45)$ & $22 / 66(33)$ \\
\hline Fiebre $\left(\mathrm{T}^{\circ} \geq 37,8^{\circ} \mathrm{C}\right)$ & $26 / 64(41)$ & $31 / 66(47)$ \\
\hline Disnea & $46 / 64(72)$ & $44 / 66(67)$ \\
\hline Taquipnea $\geq 30$ respiraciones/minuto & $27 / 59(46)$ & $35 / 65(54)$ \\
\hline Tos & $53 / 64(83)$ & $52 / 66(79)$ \\
\hline Expectoración & $47 / 64(73)$ & $41 / 66(62)$ \\
\hline Frecuencia cardíaca $\geq 125$ latidos/minuto & 9/64 (14) & $7 / 66(10)$ \\
\hline Presión arterial sistólica $<90 \mathrm{~mm} \mathrm{Hg}$ & $4 / 64 \quad(6)$ & $4 / 66 \quad(6)$ \\
\hline Estadía en el hospital, días (promedio \pm DS) & $7,7 \pm 5,8$ & $7,0 \pm 5,0$ \\
\hline Letalidad en el hospital & $4 / 64(6,3 \%)$ & $4 / 66(6,1 \%)$ \\
\hline
\end{tabular}

$* \mathrm{p}<0,05$; EPOC: enfermedad pulmonar obstructiva crónica.

Nota: El número del denominador corresponde a los pacientes con la variable registrada. 
Tabla 2. Hallazgos radiográficos y de laboratorio en 130 pacientes hospitalizados por neumonía adquirida en la comunidad

\begin{tabular}{lcc}
\hline Hallazgos & $\begin{array}{c}\text { Pacientes con etiología } \\
\text { identificada }(\mathbf{n}=\mathbf{6 4})\end{array}$ & $\begin{array}{c}\text { Pacientes sin etiología } \\
\text { identificada (n = 66) }\end{array}$ \\
\hline Radiografía de Tórax & $\mathrm{n} /$ total (\%) & $\mathrm{n} /$ total (\%) \\
Patrón de condensación & $59 / 64(92)$ & $63 / 66(95)$ \\
Compromiso multilobar o bilateral & $18 / 64(28)$ & $19 / 66(35)$ \\
Derrame pleural & $16 / 64(25)$ & $12 / 66(20)$ \\
& & \\
Laboratorio & & \\
$\mathrm{PaO}_{2}<60 \mathrm{~mm} \mathrm{Hg} \mathrm{ó} \mathrm{SaO}_{2}<90 \%$ (aire ambiental) & $41 / 63(65)$ & $44 / 66(67)$ \\
$\mathrm{Leucocitos} 10.500 \mathrm{xm} \mathrm{mm}^{3}$ & $31 / 63(49)$ & $31 / 64(48)$ \\
Nitrógeno ureico sérico $\geq 30 \mathrm{mg} / \mathrm{dl}$ & $13 / 56(23)$ & $17 / 59(29)$ \\
Sodio $<130 \mathrm{mEq} / \mathrm{L}$ & $6 / 61(10)$ & $6 / 66(9)$ \\
\hline
\end{tabular}

$\mathrm{PaO}_{2}$ : Presión parcial de oxígeno de sangre arterial

$\mathrm{SaO}_{2}$ : Saturación arterial de oxígeno

Nota: El número del denominador corresponde a los pacientes con la variable registrada.

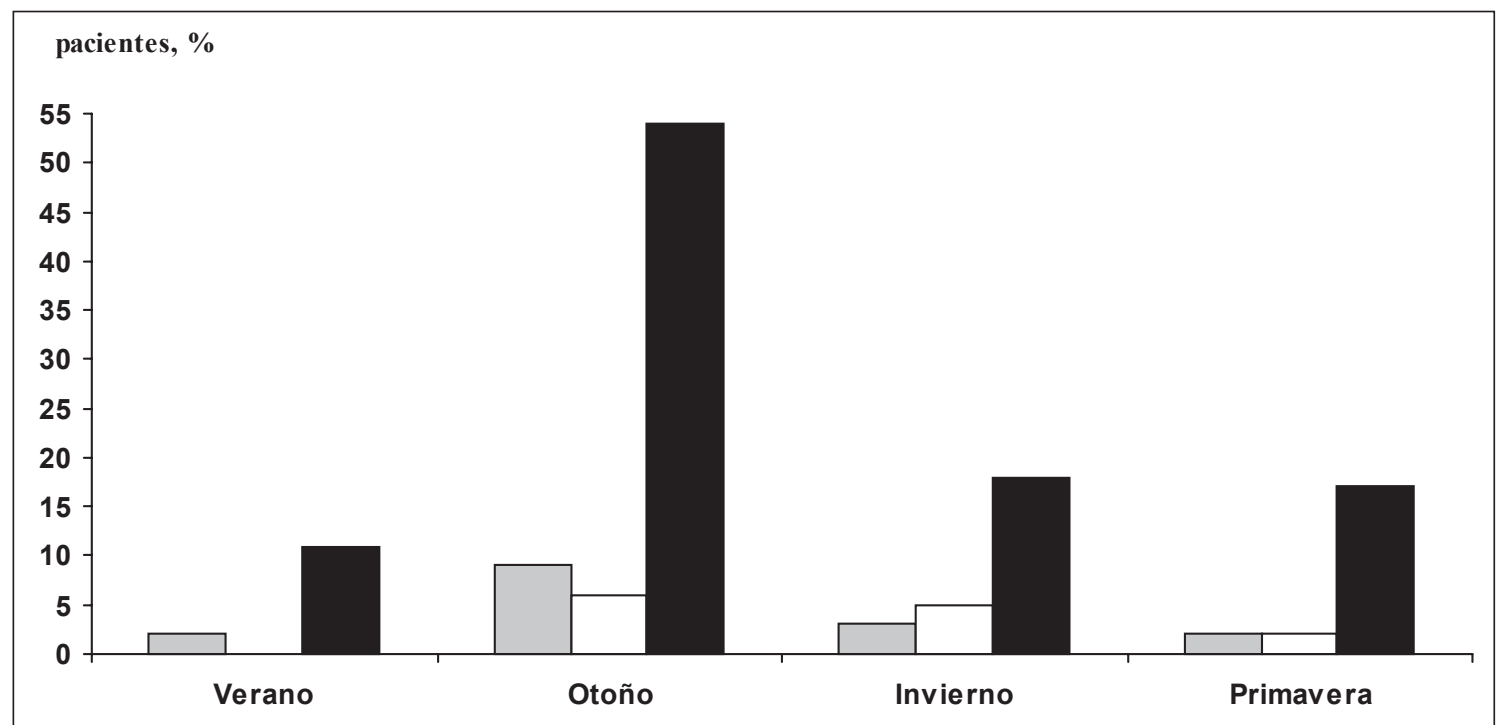

Figura 1. Distribución estacional de pacientes hospitalizados con neumonía neumocócica (gris), por virus respiratorios (blanco) y total (negro). Período de marzo de 2003 a junio de 2004, hospital Clínico Universidad Católica, Santiago, Chile.

tico antes del ingreso se aisló un microorganismo en el $45 \%$ de los casos comparado con el $49 \%$ entre los que no recibieron un antimicrobiano (p: n.s.).

En la Tabla 3 se muestran los hallazgos microbiológicos y el rendimiento de los distintos exámenes. La técnica de mayor rendimiento diagnóstico fue el panel viral (25\%) seguido por el cultivo de expectoración (24\%) y los hemocultivos (11\%). Los principales microorganismos aislados fueron $S$. pneumoniae (22/64: 34\%), Parainfluenza 1 a 3 (14/64: 22\%), Influenza A o
B (9/64: 14\%) y los bacilos Gram negativos con inclusión de $H$. influenzae (7/64: 11\%), Chlamydia pneumoniae (4/64: 6\%), Mycoplasma pneumoniae (4/64: 6\%), Adenovirus (3/64: 5\%) y Legionella pneumophila (2/64: 3\%). P. aeruginosa no fue aislada. En 13 de 121 (11\%) pacientes hemocultivados hubo bacteremia. En 12 casos el agente fue $S$. pneumoniae y en otro fue $S$. aureus. Ningún sujeto con bacteremia falleció y la edad promedio de ellos fue 59,6 $\pm 21,4$ años versus $68,8 \pm 18(\mathrm{p}=0,09)$ de los no bacterémicos, 4 ingresaron a una unidad crítica 
Tabla 3. Microorganismos aislados y rendimiento de los exámenes en 130 adultos hospitalizados por neumonía adquirida en la comunidad

\begin{tabular}{|c|c|c|c|c|c|c|}
\hline Microorganismos $^{\mathrm{C}}$ & $\begin{array}{l}\text { Esputo } \\
\text { (n) }\end{array}$ & $\begin{array}{c}\text { Hemocultivo } \\
\text { (n) }\end{array}$ & $\begin{array}{l}\text { Panel } \\
\text { viral }\end{array}$ & $\begin{array}{l}\text { Serología } \\
\text { (agudo) } \\
\text { (n) }\end{array}$ & $\begin{array}{l}\text { Serología } \\
\text { (par) } \\
\text { (n) }\end{array}$ & $\begin{array}{c}\text { Antígeno } \\
\text { urinario } \\
\text { L. pneumophila } \\
\text { (n) }\end{array}$ \\
\hline S. pneumoniae* & 8 & 12 & - & - & - & - \\
\hline H. influenzae & 3 & 0 & - & - & - & - \\
\hline K. pneumoniae & 1 & 0 & - & - & - & - \\
\hline S. aureus & 1 & 1 & - & - & - & - \\
\hline M. catarrhalis & 1 & 0 & - & - & - & - \\
\hline S. marcescens & 3 & 0 & - & - & - & - \\
\hline Influenza A & - & - & 8 & - & - & - \\
\hline Influenza B & - & - & 1 & - & - & - \\
\hline Parainfluenza 1-3 & - & - & 14 & - & - & - \\
\hline Adenovirus & - & - & 3 & - & - & - \\
\hline Virus Sincicial Respiratorio & - & - & 1 & - & - & - \\
\hline M. pneumoniae & - & - & - & 1 & 3 & - \\
\hline C. pneumoniae & - & - & - & 3 & 1 & - \\
\hline C.psittaci & - & - & - & 0 & 0 & - \\
\hline L. pneumophila & - & - & - & - & - & 2 \\
\hline Muestras positivas /muestras totales, $\mathrm{n}$ & $17 / 72$ & $13 / 121$ & $27 / 110$ & $4 / 119$ & $4 / 54$ & $2 / 115$ \\
\hline Rendimiento de los exámenes, \% & $(24)$ & $(11)$ & $(25)$ & (3) & $(7)$ & $(2)$ \\
\hline
\end{tabular}

$C_{\text {En }} 6$ pacientes se aislaron 2 ó más patógenos: $S$. pneumoniae (hemocultivo) + Parainfluenza 2 (1); S. pneumoniae (hemocultivo) + M. pneumoniae (1); S. aureus (hemocultivo) $+S$. pneumoniae (esputo) (1); Parainfluenza $2+C$. pneumoniae (1); M. pneumoniae + Virus Sincicial Respiratorio (1); Peptostreptococcus micros + Streptococcus anginosus (en líquido pleural) + Adenovirus (1).

*En otros 2 pacientes se identificó $S$. pneumoniae con el antígeno urinario.

y el promedio de estadía fue $8,8 \pm 8,3$ días. Los pacientes con neumonía neumocócica (excluyendo etiología mixta) fueron 19 y comparados con los 23 casos infectados con virus respiratorios tenían menos edad ( $59 \pm 18$ versus $72 \pm 17$ años; $p=0,021)$, menos comorbilidad (47 versus $87 \%$; $\mathrm{p}=0,0001)$, una menor proporción pertenecía a las clases de riesgo IV o V de Fine (58 versus $65 \%$; p: n.s.), ingresaron menos a unidades críticas (31 versus 39\%; p: n.s.) y la letalidad fue casi la mitad (5 versus $9 \%$; p: n.s.). Entre las 20 cepas de $S$. pneumoniae aisladas por cultivo, la resistencia a eritromicina fue $15 \%$, a penicilina 5\% (una cepa con concentración inhibitoria mínima $[\mathrm{CIM}]=2 \mathrm{mg} / \mathrm{dl})$ y $0 \%$ a cefotaxima.

Los virus respiratorios más frecuentes fueron Parainfluenza 2 (10) e Influenza A (8) y entre el conjunto de pacientes infectados con virus respiratorios (23) las enfermedades crónicas subyacentes más frecuentes fueron cardiovasculares (35\%), EPOC/asma (22\%) y diabe- tes mellitus (22\%). El 40\% había recibido la vacuna antigripal en la campaña ministerial anterior al ingreso al hospital.

El bacilo Gram negativo $S$. marcescens fue aislado en 3 pacientes, 2 de los cuales tenían marcado deterioro funcional (postrados) previo al ingreso, tuvieron una hospitalización prologada (promedio 19 días) y uno falleció. C. pneumoniae fue el más frecuente de los llamados agentes "atípicos" hallándose en 3 pacientes como único patógeno. La duración de los síntomas antes del ingreso fue prolongada (promedio 13 días), eran grandes fumadores (promedio 27 paquetes año), tenían una neumonía de alto riesgo según el índice pronóstico de Fine e ingresaron a intermedio o a UCI. Ninguno falleció y el promedio de estadía en el hospital fue 11 días.

Tratamiento antibiótico: 108 pacientes (83\%) fueron tratados con cefalosporina de tercera generación sola $(\mathrm{n}=44)$, asociada con claritromicina $(\mathrm{n}=38)$ o con una fluoroquinolona $(\mathrm{n}=26)$. Entre los 50 pacientes con un micro- 
Tabla 4. Categorías de riesgo, admisión a Unidad Crítica, estadía y letalidad en el hospital, según microorganismo aislado en 130 pacientes con neumonía adquirida en la comunidad

\begin{tabular}{|c|c|c|c|c|c|c|c|c|}
\hline \multirow[t]{2}{*}{ Microorganismo } & \multicolumn{2}{|c|}{$\begin{array}{c}\text { Total } \\
\text { Pacientes }\end{array}$} & \multirow{2}{*}{$\begin{array}{c}\text { Fallecidos } \\
\text { n }\end{array}$} & \multicolumn{2}{|c|}{$\begin{array}{c}\text { Categoría de } \\
\text { riesgo de Fine } \\
\text { IV-V }\end{array}$} & \multicolumn{2}{|c|}{$\begin{array}{c}\text { Ingreso a } \\
\text { Intermedio } \\
\text { o Intensivo }\end{array}$} & \multirow{2}{*}{$\begin{array}{c}\text { Días de } \\
\text { Hospitalización } \\
(\text { promedio } \pm \text { DS })\end{array}$} \\
\hline & $\mathbf{n}$ & $(\%)^{*}$ & & $\mathbf{n}$ & $(\%)$ & n & $(\%)$ & \\
\hline S. pneumoniae & 19 & (15) & 1 & 11 & (58) & 6 & (31) & $8,6 \pm 7,7$ \\
\hline Parainfluenza 1-3 & 12 & (9) & 1 & 8 & (67) & 3 & (25) & $5,3 \pm 2$ \\
\hline Influenza A-B & 9 & (7) & 0 & 6 & (67) & 4 & (44) & $5,3 \pm 2,6$ \\
\hline S. marcescens & 3 & (2) & 1 & 2 & (67) & 1 & (33) & $19 \pm 9$ \\
\hline H. influenzae & 3 & (2) & 0 & 1 & (33) & 0 & $(0)$ & $5 \pm 1$ \\
\hline Adenovirus & 2 & (2) & 1 & 2 & (100) & 2 & (100) & $7,5 \pm 0,7$ \\
\hline S. aureus & 1 & (1) & 0 & 1 & (100) & 0 & (0) & $9 \pm 0$ \\
\hline K. pneumoniae & 1 & (1) & 0 & 1 & (100) & 1 & (100) & $8 \pm 0$ \\
\hline M. pneumoniae & 2 & (2) & 0 & 1 & $(50)$ & 1 & $(50)$ & $9 \pm 1,4$ \\
\hline C. pneumoniae & 3 & (2) & 0 & 3 & (100) & 3 & (100) & $11,3 \pm 5,8$ \\
\hline L. pneumophila & 2 & (2) & 0 & 1 & $(50)$ & 0 & $(0)$ & $11 \pm 0$ \\
\hline M. catarrhalis & 1 & (1) & 0 & 0 & $(0)$ & 0 & (0) & $4 \pm 0$ \\
\hline Etiología mixta^ & 6 & (5) & 0 & 2 & (33) & 0 & $(0)$ & $6,5 \pm 5,3$ \\
\hline Virus respiratorios ${ }^{\mathrm{c}}$ & 23 & (18) & 2 & 16 & (70) & 9 & (39) & $5,5 \pm 2,2$ \\
\hline Agentes atípicos ${ }^{\&}$ & 7 & (5) & 1 & 5 & (71) & 4 & (57) & $10,6 \pm 3,3$ \\
\hline Bacilos Gram negativos $\$$ & 4 & (3) & 1 & 3 & (75) & 2 & (50) & $16,5 \pm 8$ \\
\hline
\end{tabular}

Unidad Crítica: Ingresos a Unidad de Cuidados Intermedios o Intensivos.

*Es el porcentaje sobre los 130 pacientes del estudio.

^Los patógenos identificados en estos pacientes están al pie de la tabla 3.

${ }^{\mathrm{C}}$ Comprende a los pacientes con virus respiratorios como agentes únicos.

${ }^{\&}$ Corresponde a la suma de pacientes con M. pneumoniae, C. pneumoniae y L. pneumophila como agentes únicos.

${ }^{\$}$ Corresponde a la suma de pacientes con $S$. marcescens y K. pneumoniae como agentes únicos.

organismo identificado y para los que existe antimicrobiano eficaz (incluyendo virus Influenza), 12 recibieron tratamiento inicial inadecuado $(24 \%)$ y uno falleció $(8,3 \%)$ comparado con 1 de los 38 pacientes $(2,6 \%)$ que recibieron tratamiento antimicrobiano adecuado (p: n.s.).

Gravedad, lugar de hospitalización y letalidad: El $82 \%$ de los pacientes pertenecía a las clases III a V de Fine y de ellos el 37\% ingresó a una UCI o a una unidad de cuidados intermedios. Entre los 85 pacientes con neumonía de alto riesgo (clases IV o V) la proporción con etiología identificada fue menor que entre los 45 de las clases de riesgo I a III (46 versus 55\%). Cuatro de 7 pacientes $(57 \%)$ con un agente atípico (L. pneumophila, M. pneumoniae o $C$. pneumoniae) y 3 de los 4 pacientes con bacilos Gram negativos (S. marcescens o K. pneumoniae) pertenecían a las clases de alto de riesgo de Fine; 4 de los 6 pacientes con etiología mixta eran de las clases de bajo riesgo y ninguno falleció. En el $48 \%$ de los pacientes ingresados a sala (88), en el $43 \%$ de los admitidos a interme- dio (23) y en el $63 \%$ de los internados en UCI (19), respectivamente, se identificó un microorganismo (p: n.s.). El promedio de hospitalización general fue 7,4 días con una mediana de 6 días y la letalidad global fue 6\%. La mitad de los fallecimientos fue dentro de la primera semana. La mortalidad y la estadía, según el agente etiológico, se muestra en la Tabla 4.

\section{DISCUSIÓN}

De acuerdo a nuestro conocimiento, esta es la primera comunicación de un estudio de la etiología de la NAC en adultos en nuestro medio incluyendo la mayoría de los patógenos relevantes y durante las 4 estaciones del año. Los principales hallazgos de este estudio son: 1) $S$. pneumoniae fue el patógeno más frecuente considerado individualmente con una moderada letalidad y resistencia a los $\beta$-lactámicos y macrólidos. 2) Los virus respiratorios y los "agentes atípicos" fueron los microorganismos que siguieron en frecuencia. 
S. pneumoniae en este estudio, tal como en series de otros países ${ }^{6-14}$ y nacionales ${ }^{2,22}$, permanece como el agente etiológico más frecuente de la neumonía comunitaria del adulto. Este hallazgo avala la guía clínica de la Sociedad Chilena de Enfermedades Respiratorias que sugiere cubrir este patógeno en todos los pacientes hospitalizados y ambulatorios con $\mathrm{NAC}^{3}$. Sin embargo, este agente no fue el más frecuente entre los pacientes con NAC grave admitidos en UCI en contraposición a lo informado en otros estu$\operatorname{dios}^{12,13}$ y en una evaluación anterior en nuestro centro $^{23}$.

La neumonía neumocócica tuvo una moderada letalidad $(5 \%)$ y entre los pacientes bacterémicos no hubo fallecidos. Esta cifra representa una diferencia importante con estudios nacionales anteriores donde la letalidad ha fluctuado entre $20^{24}$ y $30 \%{ }^{25}$ y con una serie de hace 3 años de este mismo hospital ${ }^{26}$. Estas diferencias pudieran explicarse principalmente porque: 1) Los pacientes del presente estudio eran más jóvenes y con menos comorbilidades. 2) Recibieron un manejo más agresivo, ya que casi un tercio fue admitido a UCI. 3) Se incorporó al tratamiento antibióticos de mayor espectro como las fluoroquinolonas y los macrólidos para el manejo de la NAC grave.

La resistencia de $S$. pneumoniae a penicilina fue menor que la encontrada en este centro en el período $1999-2001^{26}$ y que la informada en dos estudios nacionales anteriores ${ }^{24,27}$. No hubo resistencia a cefotaxima, mientras que la resistencia a eritromicina alcanzó el 15\%. Esta última cifra es mayor que la comunicada por Soler y $\operatorname{cols}^{27}$ y la de nuestra serie anterior en este hospita ${ }^{26}$. Un factor que podría explicar la disminución de la resistencia a la penicilina es que, debido a las medidas regulatorias para la prescripción de antibióticos del Ministerio de Salud de 1999, las ventas en farmacias de las penicilinas han bajado considerablemente; en cambio, si bien disminuyeron las ventas de eritromicina, aumentaron las de otros macrólidos (claritromicina y azitromicina) para los cuales $S$. pneumoniae tiene resistencia cruzada ${ }^{28}$.

Todos los pacientes con neumonía neumocócica recibieron tratamiento con cefalosporinas de segunda o tercera generación y ningún enfermo con S. pneumoniae resistente falleció. Este hallazgo apoya la evidencia de la literatura sobre la importancia clínica de la resistencia de $S$. pneumoniae en dos sentidos: 1) El aumento de la mortalidad de la neumonía neumocócica estaría asociado a niveles de resistencia a penicilina con CIM igual o mayor a $4 \mathrm{mg} / \mathrm{dl}$, lo cual no se ha informado en esta y otras series nacionales ${ }^{24,26,27}$. 2) La eficacia de los agentes $\beta$-lactámicos (los más utilizados en nuestra serie) para la neumonía neumocócica se ha demostrado en un estudio con un número mayor de pacientes y alta incidencia de resistencia ${ }^{29}$. El conjunto de esta información, sugiere que la importancia clínica de $S$. pneumoniae resistente a antimicrobianos en adultos con NAC en nuestro medio es aún un problema menor.

El segundo hallazgo prominente en este estudio fue que, en conjunto, los virus respiratorios siguieron en frecuencia a las bacterias como agentes etiológicos de la NAC del adulto. La frecuencia de los agentes virales en pacientes con neumonía confirmada por radiografía de tórax fluctúa entre 2 y $23 \% 0^{9,14,30-33}$. En un estudio, los virus fueron el segundo agente en frecuencia ${ }^{14}$, pero en una serie de pacientes mayores de 74 años sólo fueron encontrados en el $2 \%{ }^{30}$. Las razones que pueden explicar estas diferencias están dadas por los virus incluidos en la evaluación, las características de la población estudiada, la época de realización de la investigación, si hubo brote o epidemia en la época de estudio y las técnicas diagnósticas utilizadas. A diferencia de los estudios citados que realizaron serología para el diagnóstico virológico, en el presente utilizamos la técnica de inmunofluorescencia directa (IFD) para múltiples virus, que es un método rápido y sensible para la detección de antígenos virales. La alta sensibilidad se ha demostrado en una evaluación de 799 virus respiratorios aislados en cultivos celulares, donde sólo el 1,9\% de éstos no fue detectado por IFD ${ }^{34}$; sin embargo, la frecuencia y el papel de los agentes virales en nuestro estudio deben interpretarse con cautela debido a que puede haber una sobreestimación de estos patógenos por las siguientes razones: 1) La elevada sensibilidad del método. 2) El aislamiento de un virus en una muestra de hisopado nasofaríngeo no asegura que éste sea el agente causal o inductor de la inflamación en el parénquima pulmonar. No obstante lo anterior, la inmunofluorescencia para múltiples virus respiratorios se recomienda como la prueba de elección para la búsqueda de agentes virales en niños con neumonía ${ }^{35}$ y constituiría una herramienta diagnóstica rápida y de costo accesible para la evaluación etiológica de adultos con $\mathrm{NAC}^{5}$.

Las características clínicas principales de los pacientes con neumonía asociada a virus respiratorios en esta serie fueron: edad avanzada, alta comorbilidad (principalmente cardiovascular), el $58 \%$ tuvo una infección de alto riesgo de acuer- 
do al índice de Fine, casi el $40 \%$ de los casos fue manejado en las unidades de intermedio o intensivo y la letalidad fue $9 \%$. En un estudio reciente se demostró que la insuficiencia cardíaca es un factor de riesgo independiente para neumonía viral ${ }^{32}$. La letalidad de los pacientes de la presente serie infectados con virus respiratorios fue semejante a la de Lim y cols. $(10 \%)^{14}$, pero en otros estudios no hubo fallecidos ${ }^{31-33}$. Estos hallazgos indican que el espectro clínico y gravedad de la neumonía por estos agentes es amplia. Además, el hecho que el virus Parainfluenza fuera el microorganismo más común en los pacientes ingresados a UCI, sugiere que al contrario de lo que comúnmente se cree, los virus también deben ser buscados en los paciente graves, especialmente sin son ancianos o tienen una enfermedad cardiovascular subyacente.

Varias son las implicancias que los presentes hallazgos relacionados a la neumonía causada por agentes virales pueden tener: 1) En Chile, estos microorganismos pueden causar neumonía con un amplio espectro clínico de gravedad en adultos que requieren hospitalización. 2) La prueba de inmunofluorescencia directa para múltiples virus respiratorios es una herramienta diagnóstica útil en adultos con NAC. 3) La detección de ciertos virus en pacientes con NAC, como Influenza A o B, tiene implicancias en el manejo epidemiológico (aislamiento del paciente) y eventual uso de agentes antivirales.

La frecuencia de los agentes "atípicos" y bacilos Gram negativos fue menor, pero aunque la muestra fue pequeña, hubo fallecidos con ambos grupos de microorganismos. El tratamiento antibiótico empírico recomendado actualmente por la guía nacional cubre los bacilos Gram negativos entéricos, pero si es necesario incluir un tratamiento empírico en todos los sujetos hospitalizados para los agentes "atípicos" necesita más evaluación. La frecuencia de Chlamydia pneumoniae y Legionella pneumophila (menos del $10 \%$ ) es semejante a la comunicada en otros estudios nacionales ${ }^{17-19} \mathrm{y}$, de acuerdo al conjunto de la información brindada por estos estudios, puede plantearse que la solicitud de los exámenes serológicos y el antígeno urinario de Legionella pneumophila podrían reservarse para situaciones especiales (pacientes graves o sin respuesta al tratamiento antibiótico inicial).

Algunas limitaciones del presente estudio deben señalarse: 1) Pese al esfuerzo de los investigadores, en el $55 \%$ de los pacientes no se pudo obtener la segunda muestra para serología y en el 45\% la expectoración. 2) Algunos métodos diagnósticos como el antígeno urinario para $S$. pneumoniae o la PCR para Mycoplasma o Chlamydia no fueron incluidos limitando nuestra capacidad de identificar más pacientes con un agente etiológico. 3) El número de pacientes fue moderado limitando la fuerza de algunas conclusiones. Pese a lo anterior, el porcentaje de pacientes con etiología identificada fue semejante a otros estudios ${ }^{30,31,33}$.

En suma, sobre la base de los resultados obtenidos podemos concluir que S. pneumoniae y los virus respiratorios fueron los microorganismos etiológicos más frecuentes en adultos hospitalizados por NAC. Estos hallazgos sugieren que el tratamiento antibiótico empírico debe continuar cubriendo al neumococo y que, especialmente con un alto nivel de virus respiratorios circulantes en la comunidad, debe buscarse sistemáticamente a estos agentes en adultos con neumonía.

\section{AGRADECIMIENTOS}

Este trabajo no hubiese sido posible sin la desinteresada colaboración de auxiliares, enfermeras (os), tecnólogos médicos, alumnos y médicos de los Servicios de Urgencias, Medicina y Laboratorios Clínicos del Hospital Clínico de la Pontificia Universidad Católica de Chile. A todos ellos nuestro profundo agradecimiento.

\section{BIBLIOGRAFÍA}

1.- Ministerio de Salud de Chile: Departamento de Estadísticas e Información de Salud. http://deis.minsal.cl/ index.asp 2001

2.- SALDÍAS F, MARDÓNEZ J M, MARCHESSE M, VIVIANI P, FARÍAS G, DÍAZ A. Cuadro clínico y factores pronósticos en la neumonía adquirida en la comunidad en adultos hospitalizados. Rev Méd Chile 2002; 130: 1373-82

3.- Sociedad Chilena de Enfermedades Respiratorias. Consenso Nacional en Neumonías Adquiridas en la Comunidad en Adultos y Niños. Rev Chil Enf Respir 1999;15: 67-136.

4.- NIEDERMAN M S, MANDELL L A, ANZUETO A, BASS J B, BROUGHTON W A, CAMPBELL G D et al. Guidelines for the management of adults with community-acquired pneumonia. Diagnosis, assessment of severity, antimicrobial therapy, and prevention. Am J Respir Crit Care Med 2001; 163: 1730-54.

5.- MANDELL L A, BARTLETT J G, DOWELL S F, FILE T M Jr, MUSHER D M, WHITNEY C. Update of practice guidelines for the management of community-acquired pneumonia in immunocompetent adults. Clin Infect Dis 2003; 37: 1405-33.

6.- MACFARLANE J T, FINCH R G, WARD M J, MACRAE A D. Hospital study of adult communityacquired pneumonia. Lancet 1982; 2: 255-8. 
7.- Community-acquired pneumonia in adults in British hospitals in 1982-1983: a survey of aetiology, mortality, prognostic factors and outcome. The British Thoracic Society and the Public Health Laboratory Service. Q J Med 1987; 62: 195-220.

8.- FANG G D, FINE M, ORLOFF J, ARISUMI D, YU V L, KAPOOR W et al. New and emerging etiologies for community-acquired pneumonia with implications for therapy. A prospective multicenter study of 359 cases. Medicine (Baltimore) 1990; 69: 307-16.

9.- BLANQUER J, BLANQUER R, BORRÁS R, NAUFFAL D, MORALES P, MENÉNDEZ R et al. Aetiology of community acquired pneumonia in Valencia, Spain: a multicentre prospective study. Thorax 1991; 46: 508-11.

10.- LIEBERMAN D, SCHLAEFFER F, BOLDUR I, HOROWITZ S, FRIEDMAN M G, LEIONONEN M et al. Multiple pathogens in adult patients admitted with community-acquired pneumonia: a one year prospective study of 346 consecutive patients. Thorax 1996; 51: 179-84.

11.- NEILL A M, MARTIN I R, WEIR R, ANDERSON R, CHERESHSKY A, EPTON M J et al. Community acquired pneumonia: aetiology and usefulness of severity criteria on admission. Thorax 1996; 51: 10106.

12.- RUIZ M, EWIG S, MARCOS M A, MARTÍNEZ J A, ARANCIBIA F, MENSA J et al. Etiology of community-acquired pneumonia: impact of age, comorbidity, and severity. Am J Respir Crit Care Med 1999; 160: 397-405.

13.- LUNA C M, FAMIGLIETTI A, ABSI R, VIDELA A J, NOGUEIRA F J, FUENZALIDA A D et al. Community-acquired pneumonia: etiology, epidemiology, and outcome at a teaching hospital in Argentina. Chest 2000; 118: 1344-54.

14.- LIM W S, MACFARLANE J T, BOSWELL T C, HARRISON T G, ROSE D, LEINONEN M et al. Study of community acquired pneumonia aetiology (SCAPA) in adults admitted to hospital: implications for management guidelines. Thorax 2001; 56: 296-301.

15.- BATES J H, CAMPBELL G D, BARRON A L, MCCRACKEN G A, MORGAN P N, MOSES E B et al. Microbial etiology of acute pneumonia in hospitalized patients. Chest 1992; 101: 1005-12.

16.- SKERRETT S J. Diagnostic testing for communityacquired pneumonia. Clin Chest Med 1999; 20: 53148

17.- TRUCCO O, VICENCIO M, SALAMANCA L, OJEDA A, OYONARTE M, PRADO V. Participación de L. pneumophila en neumonía extrahospitalaria del adulto en Santiago. Rev Chil Infect 1993; 10: 89-95.

18.- LOBOS T, CARSTENS M, PFENNINGER A, MORENO R. Diagnóstico de neumonía por Legionella pneumophila mediante inmunofluorescencia directa de lavado bronquioalveolar. Rev Chil Enf Respir 1994; 10: 71-7.

19.- LOBOS T, SALDÍAS F, CARTAGENA C, JOVER E, ÁLVAREZ M, MORENO R. Chlamydia pneumoniae en pacientes con neumonías adquiridas en la comunidad en Santiago de Chile. Rev Méd Chile 1998; 126: 1483-9.

20.- FERNÁNDEZ M, ZAGOLÍN M, RUIZ M, MARTÍNEZ M A, DÍAZ J C. Neumonía adquirida en la comunidad en el paciente hospitalizado: un estudio etiológico. Rev Méd Chile 2003; 131: 498-504.

21.- FINE M J, AUBLE T E, YEALY D M, HANUSA B H, WEISSFELD L A, SINGER D E et al. A prediction rule to identify low-risk patients with communityacquired pneumonia. N Engl J Med 1997; 336: $243-$ 50.

22.- JIMÉNEZ P, SALDÍAS F, MENESES M, SILVA M E, WILSON M G, OTTH L. Diagnostic fiberoptic bronchoscopy in patients with community-acquired pneumonia. Comparison between bronchoalveolar lavage and telescoping plugged catheter cultures. Chest 1993; 103: 1023-7.

23.- DÍAZ A, ÁlVAREZ M, CALLEJAS C, ROSSO R, SCHNETTLER K, SALDÍAS F. Cuadro clínico y factores pronósticos de la neumonía comunitaria grave en adultos hospitalizados en la Unidad de Cuidados Intensivos. Arch Bronconeumol 2005 (en prensa).

24.- RIOSECO M L, RIQUELME R. Neumonía neumocócica bacterémica en 45 adultos hospitalizados. Rev Méd Chile 2004; 132: 588-94.

25.- NORIEGA L M, GONZÁLEZ P, CANALS C, MICHAUD P. Septicemia por Streptococcus pneumoniae. Análisis de 40 casos. Rev Méd Chile 1994; 122: $1385-92$.

26.- DÍAZ A, TORRES C, FLORES L J, GARCÍA P, SALDÍAS F. Neumonía neumocócica adquirida en la comunidad en adultos hospitalizados. Rev Méd Chile 2003; 131: 505-14.

27.- SOLER T, SALAMANCA L, ARBO G, MOLINA E. Susceptibilidad in vitro de cepas de Streptococcus pneumoniae aisladas de infecciones respiratorias bajas en el Instituto Nacional del Torax (INT). Rev Méd Chile 2002; 130: 304-8.

28.- BAVESTRELLO L, CABELLO A, CASANOVA D. Impacto de las medidas regulatorias en las tendencias del consumo de antibióticos en la comunidad en Chile. Rev Méd Chile 2002; 130: 1265-72.

29.- ROSÓN B, CARRATALA J, TUBAU F, DORCA J, LINARES J, PALLARES $\mathrm{R}$ et al. Usefulness of betalactam therapy for community-acquired pneumonia in the era of drug-resistant Streptococcus pneumoniae: a randomized study of amoxicillin-clavulanate and ceftriaxone. Microb Drug Resist 2001; 7: 85-96.

30.- EL-SOLH AA, SIKKA P, RAMADAN F, DAVIES J. Etiology of severe pneumonia in the very elderly. Am J Respir Crit Care Med 2001; 163: 645-51.

31.- BOCHUD P Y, MOSER F, ERARD P, VERDON F, STUDER J P, VILLARD G et al. Community-acquired pneumonia. A prospective outpatient study. Medicine (Baltimore) 2001; 80: 75-87.

32.- DE ROUX A, MARCOS M A, GARCÍA E, MENSA J, EWIG S, LODE $\mathrm{H}$ et al. Viral community-acquired pneumonia in nonimmunocompromised adults. Chest 2004; 125: 1343-51.

33.- JOKINEN C, HEISKANEN L, JUVONEN H, KALLINEN S, KLEEMOLA M, KOSKELA M et al. Microbial etiology of community-acquired pneumonia in the adult population of 4 municipalities in eastern Finland. Clin Infect Dis 2001; 32: 1141-54.

34.- LANDRY M L, FERGUSON D. SimulFluor respiratory screen for rapid detection of multiple respiratory viruses in clinical specimens by immunofluorescence staining. J Clin Microbiol 2000; 38: 708-11.

35.- MCINTOSH K. Community-acquired pneumonia in children. N Engl J Med 2002; 346: 429-37. 